\title{
APPLICATIONS OF SANDPILE ALGORITHMS TO MODELLING EDGE LOCALISED MODE PHENOMENOLOGY IN MAGNETICALLY CONFINED FUSION PLASMAS
}

\author{
CRAIG BOWIE 1
}

(Received 7 May 2021; first published online 22 June 2021)

2020 Mathematics subject classification: primary 82B27.

Keywords and phrases: critical phenomena in equilibrium statistical mechanics, fusion plasma, edge localised mode, sandpile model.

Understanding the behaviour of edge localised modes (ELMs) is of critical significance in fusion plasma research. Simple sandpile models can provide information about the behaviour of complex systems, without incorporating all of the detailed physics which may be included in a fully featured model. Here, the sandpile model developed by Chapman [6] is employed to study aspects of ELMing behaviour. Discrete waiting times between sandpile avalanches are observed to have points of similarity with discrete waiting times between ELMs in fusion plasmas. This suggests that multiple waiting times between ELMs may arise from a single cause, without the need to impute separate causes for each waiting time. Motivated by H-mode pedestals in fusion plasmas, the sandpile model is extended, using a variety of algorithms, to include a pedestal. It is observed that the behaviour of an algorithm in which the pedestal results from feedback effects most closely matches data from fusion experiments. Suppression of feedback is shown to reduce the size of the largest avalanches, and it is suggested that the same may be effective in a fusion plasma. Increases in fuelling rates are considered in a 'running model', introduced here, in which fuelling continues, rather than being paused, during systemwide avalanches. The total energy in the system reaches a local maximum when the fuelling rate is around 0.3 times the critical gradient needed to trigger an avalanche (which may be internal or systemwide). Further, it is shown that the model has an analytic solution when the fuelling rate is high enough to satisfy two necessary conditions, both of which are identified here. Motivated by pellet injection systems in fusion plasmas, both the original sandpile model, and the modified versions developed here, are then employed to test whether adding 'pellets' of sand to the sandpile can reduce the size

Thesis submitted to the Australian National University in June 2020; degree approved on 11 December 2020; supervisors Matthew Hole, Robert Dewar and Richard Dendy.

(C) 2021 Australian Mathematical Publishing Association Inc. 
of the largest avalanches. Pellets of sand added at the core in the original model are found not to be effective in reducing the size of the largest avalanches. Employing the feedback model developed here, it is observed that continuous addition of small amounts of sand, within the pedestal in the sandpile, is most effective in reducing the size of the largest avalanches. The results allow for quantitative statements about the necessary fuelling and suggest that maximal reduction of avalanche size, coupled with minimal reduction of system energy, occurs when about $2.5 \%$ of total fuelling is added at the top of the pedestal and the balance at the core. Small pellets, which quasi-continuously add particles to the sandpile (once their ablation times are taken into account), are shown to have a similar effect. It is suggested that quasi-continuous fuelling of around $2.5 \%$ of total fuelling, added at the top of the pedestal, may cause a similar reduction in the sizes of the largest ELMs in a fusion plasma. Importantly, in the model developed here, avalanche size reduction is achieved via continuous (or quasi-continuous) addition of fuel in the pedestal, rather than by synchronising pellets to waiting times between avalanches, and it is conjectured that this may also be true for a fusion plasma.

Some of this research has been published in [1-5].

\section{References}

[1] C. A. Bowie, R. O. Dendy and M. J. Hole, 'Delay time embedding of mass loss avalanches in a fusion plasma-oriented sandpile model', Phys. Plasmas 23 (2016), Article ID 100703.

[2] C. A. Bowie and M. J. Hole, 'Pedestals and feedback in fusion-plasma relevant sandpile models', Phys. Plasmas 25 (2018), Article ID 012511.

[3] C. A. Bowie and M. J. Hole, 'Behaviour of multiple discrete waiting times between mass loss events in a sandpile model', Plasma Res. Express 2 (2020), Article ID 035003.

[4] C. A. Bowie and M. J. Hole, 'Sandpile modelling of dual location fuelling in fusion plasmas', Plasma Phys. Control. Fusion 62 (2020), Article ID 055006.

[5] C. A. Bowie and M. J. Hole, 'Sandpile modelling of pellet pacing in fusion plasmas', Phys. Rev. E 101 (2020), Article ID 013207.

[6] S. C. Chapman, 'Inverse cascade avalanche model with limit cycle exhibiting period doubling, intermittency, and self-similarity', Phys. Rev. E 62 (2000), Article ID 1905.

CRAIG BOWIE, Mathematical Sciences Institute, Australian National University, Canberra, ACT 2601, Australia e-mail: craig.bowie@anu.edu.au 